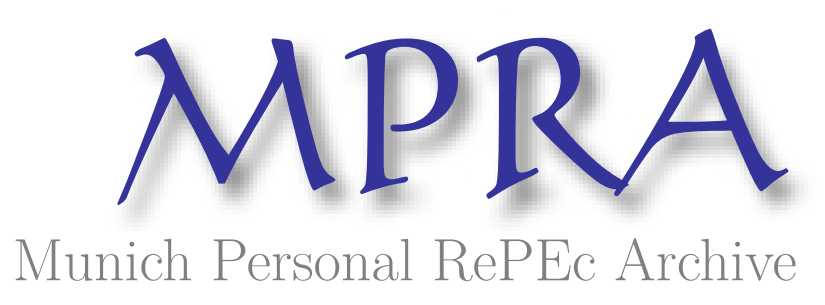

Extreme risk in Asian equity markets

Cotter, John

2007

Online at https://mpra.ub.uni-muenchen.de/3536/

MPRA Paper No. 3536, posted 13 Jun 2007 UTC 


\title{
Extreme risk in Asian equity markets
}

\author{
JOHN COTTER \\ University College Dublin
}

\begin{abstract}
Extreme price movements associated with tail returns are catastrophic for all investors and it is necessary to make accurate predictions of the severity of these events. Choosing a time frame associated with large financial booms and crises this paper investigates the tail behaviour of Asian equity market returns and quantifies two risk measures, quantiles and average losses, along with their associated average waiting periods. Extreme value theory using the Peaks over Threshold method generates the risk measures where tail returns are modelled with a fat-tailed Generalised Pareto Distribution. We find that lower tail risk measures are more severe than upper tail realisations at the lowest probability levels. Moreover, the Kuala Lumpar Composite exhibits the largest risk measures.
\end{abstract}

JEL classification: G1; G10.

Keywords: Risk measures, Asian equity markets, extreme value theory.

February 292007

Address for Correspondence:

Professor John Cotter, Centre for Financial Markets, Graduate School of Business, University College Dublin, Blackrock, Co. Dublin, Ireland.

Ph. 00-353-1-7168900

E-mail.john.cotter@ucd.ie 


\section{Introduction}

Whilst it is hard to determine the holding period of an average investor, we know that portfolio performance, and therefore the investor's outcomes, are dependent on a few days of trading. Average daily returns are near zero but we know on any one day that extreme and large scale returns can occur. Take an investment in an Asian market between 1995 and 2000 as an example, we know that returns were generally positive over most of this period but that the Asian crises over a relatively short timeframe would have reversed much of the good performance. Thus any investment strategy should incorporate adequate risk management practices to protect the overall performance of the portfolio. Investors, if they are going to manage the risk of their portfolio will try in the first instance to adequately model and measure the risks associated with their portfolio. More specifically, investors are interested in extreme risks (those that make the most impact on the bottom line performance of their portfolios) rather than average outcomes. This paper outlines and estimates two extreme risk measures for investment in six Asian markets encompassing a number of extreme price movements including the 1987 crash and the Asian crises of 1997/98.

We give two measures that estimates the risk associated with extreme price movements of a probability distribution function. We separate out the risk measures for both tails of the distribution as we know that equity returns exhibit excess skewness in general (for references see Adcock and Shutes, 2005) and distinctive tail behaviour in particular (for references see Cotter, 2001). First we use Value at Risk (VaR) that is a quantile measure that represents the maximum loss for a given probability. However, the VaR has been heavily criticised as a risk measure on the grounds that it does not satisfy the properties of coherence and, most particularly, because the VaR is not subadditive (Artzner et al. 1999; Acerbi, 2004). The failure of VaR to be subadditive can then lead to strange and undesirable outcomes: the use of the VaR takes no account of the magnitude of possible losses exceeding VaR, and can therefore leave the investor heavily exposed to very high losses exceeding the VaR. Second we use Excess Shortfall (ES) that measures the average loss if the VaR quantile is exceeded. Both of theses estimators are applied to the tails of a probability distribution as in figure 1, where the former analyses returns upto a predefined threshold or probability level, $\alpha$, and the latter is the average of the losses beyond the 
probability level, $\alpha$. The ES measure is closely related, but not identical to, the Tail Conditional Expectation, which is the probability-weighted average of losses exceeding VaR. ${ }^{1}$ Unlike the VaR, the ES is coherent (and hence subadditive as well) and so satisfies many of the properties we would desire a priori from a 'respectable' risk measure. ${ }^{2}$ The ES is bigger than the VaR and, more importantly, takes account of the magnitude of losses exceeding the VaR.

\section{INSERT FIGURE 1 HERE}

Using these risk measures we use two approaches to model the tail behaviour. First we use Extreme Value Theory (EVT) that statistically captures the tail of a distribution of returns. The literature is supportive of this approach as it adequately models the shape of financial returns and in particular the fat-tailed property associated with market outcomes spanning many different asset classes in both spot and derivative markets. ${ }^{3}$ Thus caution in using derivatives to manage the risk in the underlying market is warranted. Risk managers must not only obtain risk measures for the underlying assets but must recognise that the distributional properties of the derivative products will affect the hedging strategy to protect against extreme returns.

We benchmark the EVT approach by providing risk measures underpinned by the gaussian distribution given its prominence in the finance literature, for example in portfolio theory with mean-variance analysis. Studies have shown that the gaussian distribution is relatively thin-tailed relative to the empirical findings for market returns leading to an underestimation of associated risk measures.

\footnotetext{
${ }^{1}$ For more on these risk measures and their distinguishing features, see Acerbi and Tasche (2001) or Acerbi (2004). We don't consider the TCE further in this paper because it is equivalent to the ES where the density function is continuous, and where it differs from the ES, it is not coherent.

${ }^{2}$ Loosely speaking, let $X$ and $Y$ represent any two portfolios' $\mathrm{P} / \mathrm{Ls}$ over a given forecast horizon, and let $\rho($.$) be a measure of risk. The risk measure \rho($.$) is subadditive if it satisfies$ $\rho(X+Y) \leq \rho(X)+\rho(Y)$. Subadditivity is the most important criterion we would expect a 'respectable' risk measure to satisfy. It can be demonstrated that VaR is not subadditive unless we impose the empirically implausible requirement that returns are elliptically distributed. Given the importance of subadditivity, the VaR's non-subadditivity makes it very difficult to regard the VaR as a 'respectable' measure of risk.

${ }^{3}$ The literature is extensive giving first support for fat-tailed market returns, for example currencies and equities, and second advocating the use of EVT in modelling this feature (Koedijk and Kool, 1994; Longin, 1996; and Cotter and Dowd, 2006; to name but a few).
} 
The outline of the paper is as follows. In the next section we describe the theoretical framework of EVT and the risk measures that are estimated. This is followed by a discussion of the preliminary statistics of the Asian markets chosen in section 3. These are equity indexes from Japan, Hong Kong, Singapore, Thailand, Indonesia and Malaysia. The first three are leading Asian markets and the latter three are the markets that were most affected by the Asian crises of 1997/98. Next, our empirical findings detail the modelling of tail behaviour using EVT on the equity data coupled with a presentation and discussion of our extreme risk measures. Finally a summary is given in section 5 .

\section{Theoretical framework and risk measures}

We begin by discussing our use of Extreme Value Theory to model the tail returns and then show how our EVT estimates are incorporated into the risk measures. Many studies have examined the modelling of tail returns. Given the existence of fat-tails, the EVT approach is supported to model tail returns in an unconditional setting. EVT analyses tail outcomes only and allows for three separate classifications of the tail distributions. We apply the Peaks over Threshold (POT) approach based on the Generalized Pareto distribution (GPD) to describe tail behaviour. ${ }^{4}$ Begin by assuming that equity returns represent the realisations of a random variable $X$ over a high threshold $u$. More particularly, if $X$ has the distribution function $F(x)$, we are interested in the distribution function $F_{u}(x)$ of exceedances of $X$ over a high threshold $u$ :

$$
F_{u}(x)=P\{X-u \leq x \mid X>u\}=\frac{F(x+u)-F(u)}{1-F(u)}
$$

As $u$ gets large which happens as you move further and further the tail, then the distribution of exceedances tends to a GPD:

\footnotetext{
${ }^{4}$ Alternatively, extreme tail returns could be modelled parametrically by Generalised Extreme Value (GEV) theory or using semi parametric methods and related estimators such as the Hill or Picklands estimators. Asymptotically the approaches are analogous. We prefer to use the parametric POT approach over semi parametric estimators; and in comparison to the GEV approach it (generally) uses one less parameter, and because the GEV approach does not utilise all extreme returns if extremes occur in clusters. We present only salient features of the literature and for a comprehensive discussion see Embrechts et al (1997).
} 


$$
G_{\xi, \beta}(x)=\left\{\begin{array}{lll}
1-(1+\xi x / \beta)^{-1 / \xi} & & \xi \geq 0 \\
1-\exp (-x / \beta) & \text { if } \quad \begin{array}{l}
\xi<0
\end{array}
\end{array}\right.
$$

where

$x \in\left\{\begin{array}{lll}{[0, \infty)} & & \xi \geq 0 \\ {[0,-\beta / \xi]} & \text { if } & \xi<0\end{array}\right.$

and the shape $\xi$ and scale $\beta>0$ parameters are estimated conditional on the threshold $u$ (Balkema and de Haan (1974); Embrechts et al., 1997, pp. 162-164).

Note that the shape parameter $\xi$ sometimes appears in GPD discussions couched in terms of its inverse, a tail index parameter $\alpha$ given by $\alpha=1 / \xi$. One advantage of EVT is that we can distinguish between different types of tail behaviour based on parameter values and in this sense the shape parameter is especially important. Thus a negative $\xi$ is associated with very thin-tailed distributions that are rarely of relevance to financial returns, and a zero $\xi$ is associated with other thin tailed distributions such as the normal. However, the most relevant for our purposes are heavy-tailed distributions associated with $\xi 0$. Market returns such as equity indexes indicate this property. The tails of such distributions decay slowly and follow a 'power law' function. Moreover the number of finite moments is ascertained by the value of $\xi$ : if $0.25 \leq \xi \leq 0.5$ (or, equivalently, $\alpha \geq 2$ ) we have infinite second and higher moments; if $\xi \leq 0.25$ (or $\alpha \geq 4$ ), we have infinite fourth and higher moments, and so forth. $\alpha$ thus indicates the number of finite moments. Evidence generally suggests that the second moment is probably finite, but the fourth moment is more problematic (see, e.g., Loretan and Phillips, 1994).

The GPD parameters can be estimated by maximum likelihood methods. The $\log$ likelihood function of the GPD for $\xi \neq 0$ is:

$$
l(\xi, \beta)=-n\left(\ln (\beta)-(1+1 / \xi) \sum_{i=1}^{n} \ln \left(1+\xi x_{i} / \beta\right)\right.
$$


where $x_{i}$ satisfies the constraints specified for $x$. If $\xi=0$, the log likelihood function is:

$$
l(\beta)=-n\left(\ln (\beta)-\beta^{-1} \sum_{i=1}^{n} x_{i}\right.
$$

ML estimates are then found by maximising the log-likelihood function using suitable (e.g., numerical optimisation) methods.

Assuming that $u$ is sufficiently high, the distribution function for exceedances is given by:

$$
F_{u}(x)=1-\frac{N_{u}}{n}\left(1+\xi \frac{x-u}{\beta}\right)^{\frac{-1}{\xi}}
$$

where $n$ the sample size and $N_{u}$ is the number of observations in excess of the threshold (Embrechts et al., 1997, p. 354).

Our risk measures are directly obtained from the distribution of exceedences. First looking at the quantile or VaR that represents a loss for a given low probability level, $\alpha$ :

$$
\operatorname{Var}_{\alpha}=\text { quantile }_{\alpha}=q_{\alpha}
$$

where $q_{\alpha}$ is the relevant quantile of the probability distribution. All returns beyond the threshold are irrelevant. This quantile is tail dependent so we calculate it separately for upper and lower tail realisations representing long and short trading positions respectively. Second, the average loss which is the average of the losses beyond the low probability level, $\alpha$. In the case of a continuous distribution, the average loss is given by:

$$
E S_{\alpha}=\text { averageloss } \alpha=\frac{1}{1-\alpha} \int_{\alpha}^{1} q_{p} d p
$$


Using the average loss measure implies taking an average of quantiles beyond the threshold in which tail quantiles have an equal weight and non-tail quantiles have a zero weight.

In order to estimate these risk measures we return to our tail return distribution of exceedences. Taking the $p^{\text {th }}$ quantile of this distribution - which is also the VaR at the (high) confidence level $p$ - is then be obtained by inverting the distribution function and adjusting for the mean return, $u$ :

$$
q_{p}=\operatorname{VaR}_{p}=u+\frac{\beta}{\xi}\left\{\left(\frac{n}{N_{u}} p\right)^{-\xi}-1\right\}
$$

The average loss is then given by:

$$
\operatorname{averageloss}_{p}=E S_{p}=\frac{q_{p}}{1-\xi}+\frac{\beta-\xi u}{1-\xi}
$$

We now turn our attention to the application of the modelling approach and the associated risk measures. First we provide some preliminary details of the Asian markets chosen for analysis.

\section{Data Description}

Daily log returns of Asian equity market indices are analysed between January 1985 and December 2000 enveloping the Asian crises. The indices chosen and their abbreviations are Nikkei 225 Stock Average (Nikkei), Hang Seng (Hang Seng), Singapore Straits Times (Singapore), Bangkok S.E.T. (Bangkok), Jakartha SE Composite (Jakartha), and Kuala Lumpar Composite (Kuala Lumpar). Time series plots of the returns series is provided in figure 2 displaying volatility clustering and the existence of large spikes (extreme returns). These extreme returns incorporate financial crises such as the October 1987 crash, the Asian crises in October 1997 and the increased (mostly downside) volatility in 2000. In general the magnitude of the 
extreme negative returns tend to be larger than positive ones, except for the Jakartha index suggesting that the lower tail risk measures may also be larger.

INSERT FIGURE 2 HERE

Further characteristics of the returns series are provided by the summary statistics in table 1. First moment values indicate a positive returns series, although approximately zero, and second moment values suggest daily volatility in excess of 1 per cent. The Nikkei exhibits the lowest levels of average unconditional risk whereas both Jakartha and Kuala Lumpar have the highest standard deviations. Very long tails both for upside and downside distributions are evident for all series from MinDev (MaxDev) that counts the number of standard deviations minimum (maximum) observed returns are from the mean. These statistics indicate that the empirical returns are associated with a longer negative tail than a positive one with the exception of the Jakartha index whose maximum return is 23.99 standard deviations above the mean. ${ }^{5}$ All series exhibit excess skewness, excess kurtosis and are non-normal for the JarqueBera test. Q-Q plots of the observed distributions against the normal distribution are presented in figure 3 indicating the magnitude of extreme values located at the tails of the distribution. The series are characterised by a fat-tail property where in comparison gaussianity underestimates the weights of the respective empirical tails and would underestimate the likelihood of extreme returns occurring.

INSERT TABLE 1 HERE

INSERT FIGURE 3 HERE

\section{Emprical findings}

As stated we fit a GPD to our equity index returns series and use these to generate the associated risk measures. The GPD parameters are given in table 2 for both tails of the distribution. A key issue is to determine where the respective tails begin. We use a number of methods that first identify possible threshold values, and then, after fitting the GPD, determine their relative goodness of fit. The threshold values are identified using the QQ plots where the point where deviations from the normal begin are natural threshold values. After fitting the GPD we develop tail plots that determine the goodness of fit from fitting the GPD and an example for the Nikkei is

\footnotetext{
${ }^{5}$ The findings for the Indonesian market are affected by the deregulation that took place at the end of 1988 where a single days return was in excess of +40 per cent.
} 
given in figure 4. This shows that the lower tail threshold of 1.5 and the upper tail threshold of 2.3 imply a good fit for the GPD as the tail plot is linear around these thresholds. $^{6}$ Also note that the threshold size impacts the number of exceedences where the higher the threshold is associated with smaller numbers of exceedences.

\section{INSERT TABLE 2 HERE}

\section{INSERT FIGURE 4 HERE}

Turning to the GPD parameters, the findings are in line with previous studies on equity markets. The tail indices are positive in support of the fat-tailed property, and the scale parameters are approximately one. There are only two exceptions where the tail index is significantly positive and this occurs for the lower tail of the Nikkei and the upper tail of the Jakartha index. The fattest tail in density mass is recorded for the Singapore index on a long position and the Kuala Lumpar index on a short position. In general the magnitude of the tail parameters support the existence of a second moment but not necessarily the fourth moment. As stated these GPD parameters feed directly into our risk measures and we now turn to this discussion.

The tail risk measures are given in table 3. Two separate risk measures are given: the tail quantiles that give the loss levels at certain probability levels and the average losses that represent the mean tail losses once the tail quantiles have been exceeded. Obviously investors would be interested in both measures. The results represent risk measures for very low probability levels. These probability levels have associated waiting periods of 100 days, 1000 days, and 10000 days measuring intervals of approximately half a year, four years and forty years respectively. For example, the loss of 9.56 per cent is recorded for the 99.99 per cent probability level for the Nikkei index and this should only be exceeded on a single day's return once every forty years on average. Both EVT estimates and gaussian estimates are presented for comparison. The key feature of equity returns, namely, the fat-tailed property is embedded in the EVT measures and is overlooked by their gaussian counterparts. Hence the extreme value estimates dwarf gaussian measures and especially at very low probability levels that correspond to longer trading intervals.

\footnotetext{
${ }^{6}$ Similar findings occur for the other markets. The remaining plots are not included for conciseness and are available on request.
} 


\section{INSERT TABLE 3 HERE}

First looking at the quantiles the findings indicate that Nikkei is the safest market and Kuala Lumpar exhibits the most extreme returns. The profile of the average losses is very similar to the quantiles. The EVT estimates are reasonably similar across trading position with the largest quantile occurring for lower tails realisations on the Singapore index and for upper tail returns on the Kuala Lumpar index. For example, the extreme risk of the Kuala Lumpar index is more than twice that of the Nikkei. For the EVT estimates there is substantial but not consistent deviations in the risk measures for long and short trading positions. For example, at the 99 per cent level the lower tail measures tend to be larger than upper tail values, but this trend reverses when we focus on the lower probability levels. Moreover the deviations from long to short positions tend to widen for lower probability levels. In contrast, the gaussian estimates remain near symmetrical and are dependent on the relatively small daily average returns. Note also that our confidence in the findings vary considerably for the extreme value findings but less so for the gaussian ones. Whilst all standard errors tend to increase for larger risk measures, this is particularly so for the extreme value estimates. Thus the lowest level of precision is recorded for the average loss on the Kuala Lumpar index at the 99.99 per cent level and on a short trading position. Precision levels in the gaussian estimates are much narrower.

\section{Summary}

This paper examines extreme risk in Asian markets. Overall portfolio performance is driven by a few exceptional trading days that dwarf the outcomes over much of the remaining trading period. These exceptional trading days give rise to tail returns and it is these that are of interest in this paper. Two statistical approaches are applied: Extreme Value Theory that models tail returns only and the gaussian distribution that underpins much of financial modelling. Two separate risk measures are developed using the two modelling approaches. First, a quantile based measure akin to the popular Value at Risk estimate that measures the loss upto a certain predefined threshold or probabilitiy level. Second, average losses that measure the mean of the losses beyond the quantile is presented. 
We first identify the fat-tailed property of the Asian equity indexes. Using a Peaks over Threshold extreme value approach we find that the Asian tail returns are adequately modelled with the fat-tailed Generalised Pareto Distribution. Our risk measures are much smaller if we apply the relatively thin tailed gaussian distribution compared to the GPD estimates. However the precision of our estimates indicates a greater level of variability for the GPD estimates that is understandable given the size of the risk measures. There is no consistency as to whether lower tail risk measures are systematically greater than upper tail ones. We identify the Kuala Lumpar as being prone to the most extreme returns giving the largest risk measures, in contrast to the relative safe Japanese market.

The findings have implications for the use of derivatives in managing the exposure resulting from extreme risk. Previous studies have found that derivative contracts are fat-tailed and adequately modelled with EVT methods. Here we document the fat tailed property and model it for Asian indexes on the underlying markets. Thus any hedging strategy seeking to protect against extreme movements in the underlying markets should incorporate methodologies that do not assume that returns belong to a gaussian distribution. Thus a non-linear approach to deriving the hedging strategy that would incorporate fat-tails would result in a more appropriate hedge against extreme returns.

\section{References:}

Acerbi, C., and D. Tasche, 2001, Expected shortfall: a natural alternative to value at risk, Economic Notes, 31, 379-388.

Acerbi, C., 2004, Coherent representations of subjective risk-aversion, pp. 147-207 in Risk Measures for the $21^{\text {st }}$ Century, G. Szego (Ed), New York: Wiley.

Adcock, C.J. and K. Shutes, An analysis of skewness and skewness persistence in three emerging markets, Emerging Market Review, 6, 396-418.

Artzner, P., F. Delbaen, J.-M. Eber, and D. Heath, 1999, Coherent measures of risk, Mathematical Finance 9, 203-228.

Balkema, A. A., de Haan, L., 1974, Residual lifetime at great age. Annals of Probability 2, 792-804.

Cotter, J., 2001, Margin Exceedences for European Stock Index Futures using Extreme Value Theory, Journal of Banking and Finance, 25, 1475-1502.

Cotter, J., and K. Dowd, 2006, Extreme Spectral Risk Measures: An Application to Futures Clearinghouse Margin Requirements, Journal of Banking and Finance, 30, 34693485 . 
Embrechts, P., Kluppelberg, C., and Mikosch, T., 1997, Modelling extremal events, (Springer, Berlin).

Koedijk, K. G. and C. J. M. Kool, 1994. Tail Estimates and the EMS Target Zone, Review of International Economics, 2: 153-165.

Longin, F.M, 1996, The asymptotic distribution of extreme stock market returns, Journal of Business, 63, 383-408.

Loretan, M. and Phillips, P. C. B., 1994. Testing the Covariance Stationarity of Heavytailed Time Series, Journal of Empirical Finance, 1, 211-248. 
Table 1. Summary statistics for daily returns series

\begin{tabular}{lrrrrrrr}
\hline \multicolumn{1}{c}{ Index } & Mean & Std D & MinDev & MaxDev & Skew & Kurt & \multicolumn{1}{c}{ J-B } \\
\hline Nikkei & 0.004 & 1.34 & -12.04 & 9.28 & -0.17 & 13.01 & 17453 \\
Hang Seng & 0.061 & 1.79 & -22.71 & 9.61 & -3.56 & 81.66 & 1084852 \\
Singapore & 0.027 & 1.45 & -20.18 & 10.68 & -2.11 & 57.05 & 510688 \\
Bangkok & 0.015 & 1.71 & -5.87 & 6.62 & 0.12 & 8.95 & 6177 \\
Jakartha & 0.044 & 1.68 & -13.45 & 23.99 & 3.97 & 110 & 2003473 \\
Kuala Lumpar & 0.019 & 1.71 & -14.17 & 12.19 & -0.26 & 34.6 & 173703 \\
\hline
\end{tabular}

Notes: The mean, standard deviation (Std D) are presented in percentages. The number of standard deviations the minimum return (MinDev) and the maximum return (MaxDev) are from the mean exhibit the length of the empirical distribution. The skewness (Skew) statistic and kurtosis (Kurt) for a normal distribution have values of 0 and 3 respectfully. Normality is examined with the Jarque-Bera (J-B) test which a critical value of 3.84. All skewness, kurtosis and normality coefficients are significant at the 5 percent level. 
Table 2. GPD parameter estimates for daily returns series

\begin{tabular}{|c|c|c|c|c|c|c|c|c|}
\hline Index & Tail & Beta & Threshold & Exceedences & Tail & Beta & Threshold & Exceedences \\
\hline & \multicolumn{2}{|c|}{ Lower Tail } & & & \multicolumn{2}{|c|}{ Upper Tail } & & \\
\hline \multirow[t]{2}{*}{ Nikkei } & 0.06 & 0.96 & 1.50 & 399.00 & 0.14 & 0.85 & 2.30 & 375.00 \\
\hline & $(0.04)$ & $(0.06)$ & & & $(0.06)$ & $(0.07)$ & & \\
\hline \multirow[t]{2}{*}{ Hang Seng } & 0.34 & 1.07 & 2.20 & 251.00 & 0.24 & 1.10 & 3.00 & 127.00 \\
\hline & $(0.08)$ & $(0.11)$ & & & $(0.11)$ & $(0.16)$ & & \\
\hline \multirow[t]{2}{*}{ Singapore } & 0.48 & 0.93 & 2.50 & 115.00 & 0.31 & 1.03 & 3.00 & 77.00 \\
\hline & $(0.13)$ & $(0.14)$ & & & $(0.14)$ & $(0.19)$ & & \\
\hline \multirow[t]{2}{*}{ Bangkok } & 0.11 & 1.15 & 1.30 & 625.00 & 0.16 & 1.17 & 1.60 & 470.00 \\
\hline & $(0.05)$ & $(0.07)$ & & & $(0.06)$ & $(0.09)$ & & \\
\hline \multirow[t]{2}{*}{ Jakartha } & 0.27 & 1.22 & 2.00 & 192.00 & 0.17 & 0.91 & 2.20 & 176.00 \\
\hline & $(0.09)$ & $(0.14)$ & & & $(0.09)$ & $(0.10)$ & & \\
\hline \multirow[t]{2}{*}{ Kuala Lumpar } & 0.29 & 1.15 & 2.00 & 255.00 & 0.40 & 1.01 & 2.40 & 187.00 \\
\hline & $(0.07)$ & $(0.11)$ & & & $(0.10)$ & $(0.12)$ & & \\
\hline
\end{tabular}

Notes: Extreme value parameters, the tail index (tail) and scale (beta) are estimated via maximum likelihood methods with standerd errors in parentheses. The number of exceedences and the associated threshold values are also given. 
Table 3. Extreme risk measures of Asian markets

\begin{tabular}{|c|c|c|c|c|c|c|c|}
\hline Index & & Q.99 & Q.999 & Q.9999 & Q.99 & Q.999 & Q.9999 \\
\hline QUANTILE & & Lower Tail & & & Upper Tail & & \\
\hline \multirow[t]{4}{*}{ Nikkei } & Extreme value & 3.81 & 6.50 & 9.56 & 3.70 & 6.90 & 11.36 \\
\hline & & $(0.10)$ & $(0.31)$ & $(0.70)$ & $(0.11)$ & $(0.46)$ & (1.28) \\
\hline & Gaussian & 3.11 & 4.14 & 4.98 & 3.12 & 4.14 & 4.99 \\
\hline & & $(0.05)$ & $(0.08)$ & $(0.10)$ & $(0.05)$ & $(0.08)$ & $(0.10)$ \\
\hline \multirow[t]{4}{*}{ Hang Seng } & Extreme value & 4.84 & 11.75 & 26.94 & 4.40 & 8.81 & 16.44 \\
\hline & & $(0.19)$ & $(1.14)$ & (4.61) & $(0.13)$ & $(0.68)$ & (2.48) \\
\hline & Gaussian & 4.10 & 5.47 & 6.59 & 4.22 & 5.59 & 6.71 \\
\hline & & $(0.06)$ & (0.09) & $(0.11)$ & $(0.06)$ & $(0.09)$ & $(0.11)$ \\
\hline \multirow[t]{4}{*}{ Singapore } & Extreme value & 3.72 & 10.10 & 29.38 & 3.70 & 7.91 & 16.56 \\
\hline & & $(0.13)$ & (1.14) & (6.67) & $(0.08)$ & $(0.67)$ & (2.88) \\
\hline & Gaussian & 3.34 & 4.45 & 5.36 & 3.39 & 4.50 & 5.41 \\
\hline & & $(0.05)$ & $(0.08)$ & $(0.10)$ & $(0.05)$ & $(0.08)$ & $(0.10)$ \\
\hline \multirow[t]{4}{*}{ Bangkok } & Extreme value & 4.91 & 8.94 & 14.11 & 5.07 & 9.89 & 16.86 \\
\hline & & $(0.15)$ & $(0.56)$ & (1.45) & $(0.17)$ & $(0.73)$ & (2.12) \\
\hline & Gaussian & 3.97 & 5.28 & 6.35 & 4.00 & 5.31 & 6.38 \\
\hline & & $(0.06)$ & $(0.09)$ & $(0.11)$ & $(0.06)$ & $(0.09)$ & $(0.11)$ \\
\hline \multirow[t]{4}{*}{ Jakartha } & Extreme value & 4.31 & 10.20 & 21.14 & 3.68 & 6.94 & 11.72 \\
\hline & & $(0.18)$ & (0.93) & $(3.46)$ & $(0.11)$ & $(0.47)$ & (1.48) \\
\hline & Gaussian & 3.86 & 5.14 & 6.29 & 3.95 & 5.23 & 6.29 \\
\hline & & $(0.06$ & (0.08 & $(0.11$ & $(0.06)$ & $(0.09)$ & (0.11) \\
\hline \multirow[t]{4}{*}{ Kuala Lumpar } & Extreme value & 4.75 & 11.14 & 23.64 & 4.46 & 11.33 & 28.46 \\
\hline & & $(0.19)$ & $(1.00)$ & (3.72) & $(0.17)$ & $(1.18)$ & (5.68) \\
\hline & Gaussian & 3.95 & 5.25 & 6.33 & 3.99 & 5.29 & 6.37 \\
\hline & & $(0.06)$ & $(0.09)$ & $(0.11)$ & $(0.06)$ & $(0.09)$ & $(0.11)$ \\
\hline AVERAGE LOSS & & Lower Tail & & & Upper Tail & & \\
\hline \multirow[t]{4}{*}{ Nikkei } & Extreme value & 4.97 & 7.82 & 11.06 & 5.07 & 8.81 & 14.01 \\
\hline & & $(0.19)$ & $(0.47)$ & $(0.95)$ & $(0.24)$ & $(0.79)$ & (1.93) \\
\hline & Gaussian & 3.57 & 4.51 & 5.30 & 3.57 & 4.51 & 5.31 \\
\hline & & $(0.06)$ & $(0.08)$ & $(0.10)$ & $(0.06)$ & $(0.08)$ & $(0.10)$ \\
\hline \multirow[t]{4}{*}{ Hang Seng } & Extreme value & 7.84 & 18.34 & 41.43 & 6.29 & 12.07 & 22.10 \\
\hline & & $(0.57)$ & (2.62) & $(9.05)$ & $(0.33)$ & $(1.43)$ & (4.33) \\
\hline & Gaussian & 4.72 & 5.97 & 7.02 & 4.83 & 6.08 & 7.14 \\
\hline & & $(0.06)$ & $(0.09)$ & $(0.12)$ & $(0.07)$ & $(0.09)$ & $(0.12)$ \\
\hline \multirow[t]{4}{*}{ Singapore } & Extreme value & 6.63 & 18.91 & 56.02 & 5.52 & 11.65 & 24.21 \\
\hline & & $(0.48)$ & (3.74) & (16.86) & $(0.33)$ & $(1.60)$ & $(5.58)$ \\
\hline & Gaussian & 3.84 & 4.85 & 5.71 & 3.88 & 4.90 & 5.76 \\
\hline & & $(0.06)$ & $(0.08)$ & $(0.10)$ & $(0.06)$ & $(0.08)$ & $(0.10)$ \\
\hline \multirow[t]{4}{*}{ Bangkok } & Extreme value & 6.64 & 11.16 & 16.96 & 7.13 & 12.87 & 21.18 \\
\hline & & $(0.31)$ & $(0.93)$ & $(2.10)$ & $(0.38)$ & (1.32) & (3.26) \\
\hline & Gaussian & 4.55 & 5.75 & 6.76 & 4.58 & 5.78 & 6.79 \\
\hline & & $(0.06)$ & (0.09) & $(0.11)$ & $(0.06)$ & $(0.09)$ & $(0.11)$ \\
\hline \multirow[t]{4}{*}{ Jakartha } & Extreme value & 6.84 & 14.89 & 29.85 & 5.08 & 8.98 & 14.72 \\
\hline & & $(0.48)$ & (1.99) & (6.17) & $(0.25)$ & $(0.89)$ & (2.35) \\
\hline & Gaussian & 4.44 & 5.61 & 0.04 & 4.52 & 5.69 & 6.69 \\
\hline & & $(0.06)$ & (0.09) & $(0.01)$ & $(0.06)$ & $(0.09)$ & $(0.11)$ \\
\hline \multirow[t]{3}{*}{ Kuala Lumpar } & Extreme value & 7.50 & 16.52 & 34.14 & 7.49 & 18.88 & 47.26 \\
\hline & & $(0.52)$ & $(2.15)$ & (6.79) & $(0.62)$ & (3.19) & (12.16) \\
\hline & Gaussian & 4.53 & 5.73 & 6.74 & 4.57 & 5.77 & 6.77 \\
\hline
\end{tabular}


\begin{tabular}{lll|ll}
$(0.06)$ & $(0.09)$ & $(0.11)$ & $(0.06)$ & $(0.09)$
\end{tabular} $(0.11)$

Notes: the statistical approaches of EVT and the gaussian distribution and related risk

measures of quantiles and average losses are described in the text. 


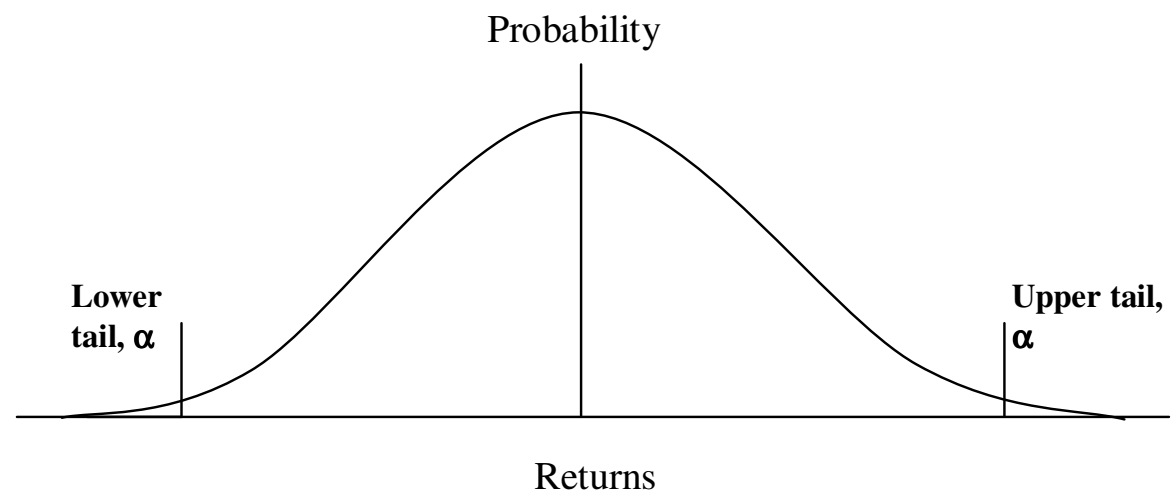

Figure 1. Lower and upper tail extreme return for a distribution of market returns Notes: This figure illustrates a distribution of index returns with high $\alpha$ threshold levels. At each tail of the distribution, a threshold is identified representing the quantile risk measure. The average of price movements beyond this threshold represents the average loss risk measure. 

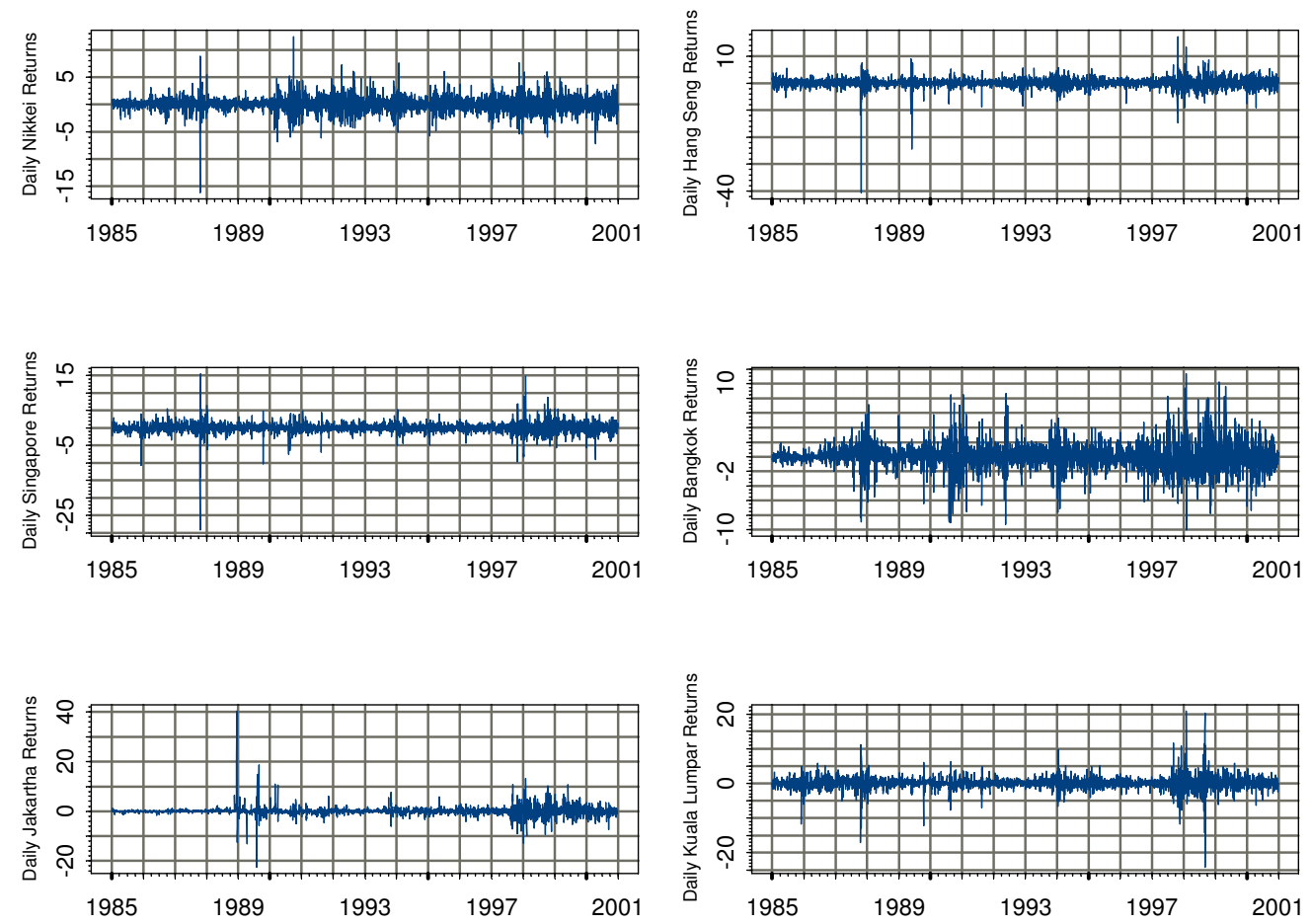

Figure 2. Time series plots of Asian index returns.

Notes: This figure shows a time series plot of daily percentage returns between January 1985 and December 2000. 

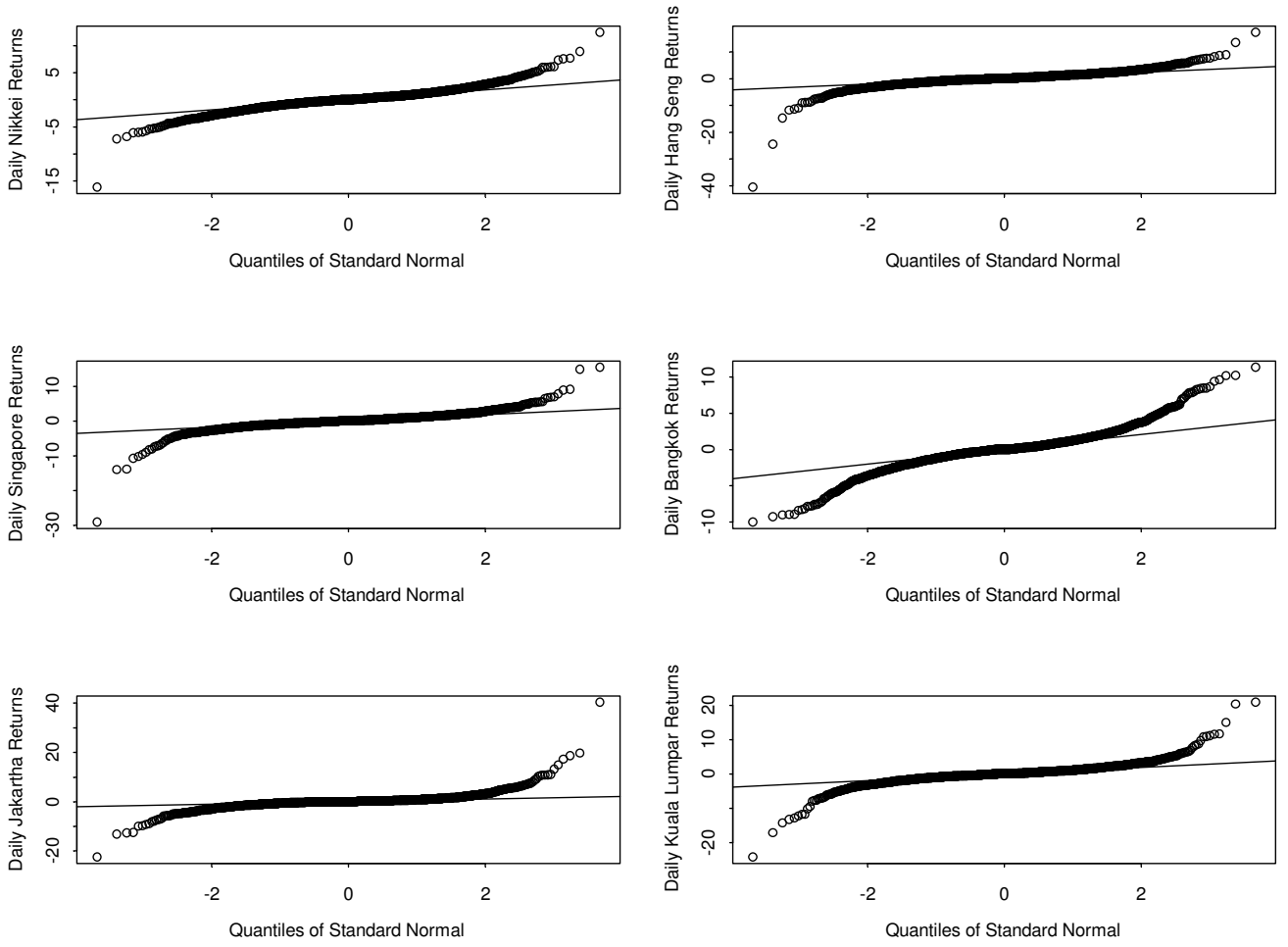

Figure 3. Q-Q plots of Asian index returns.

This figure plots the quantiles of the observed distribution against the normal distribution (straight line) for the daily series. 

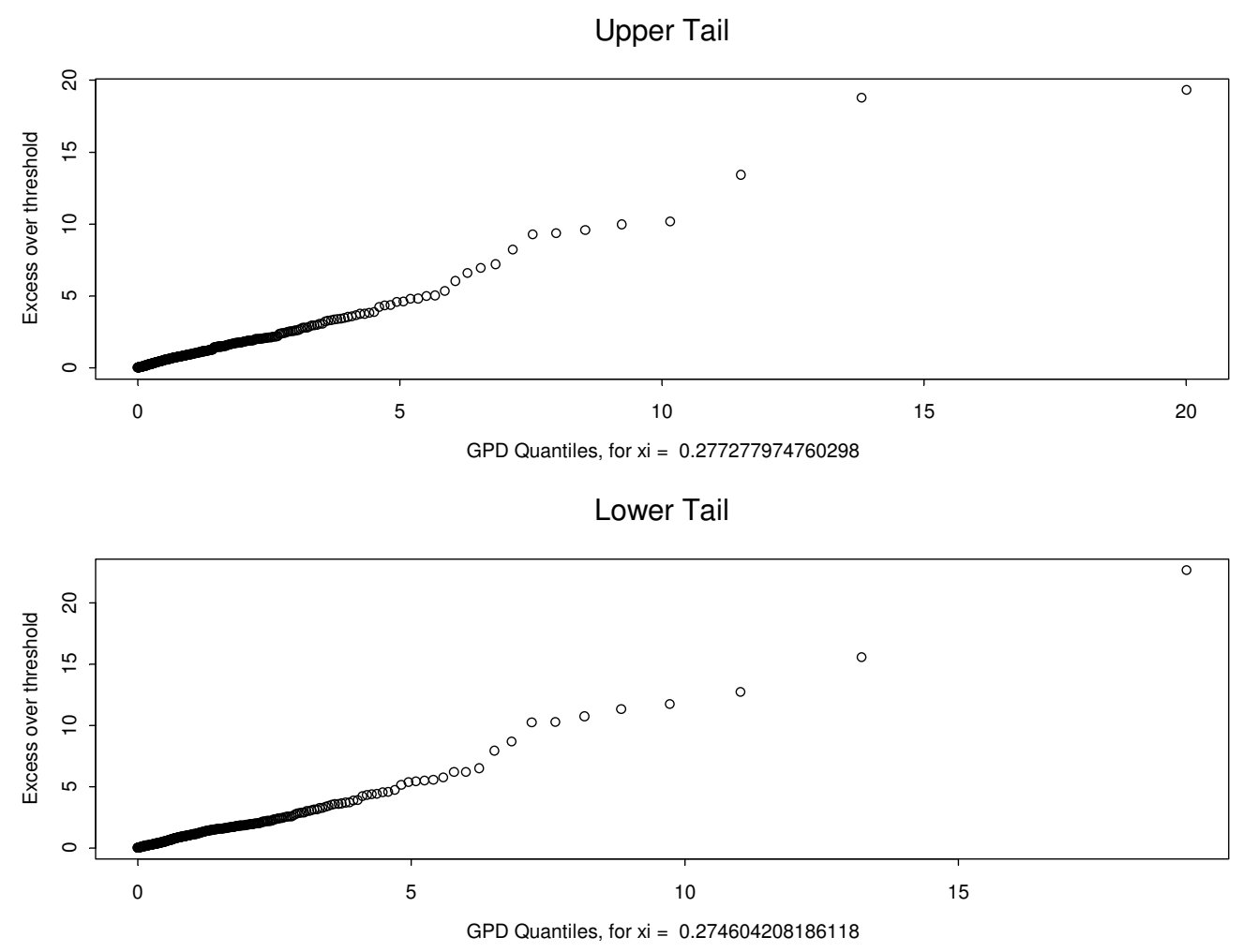

Figure 4. Tail plot of Nikkei index returns.

Notes: this plot shows the tail realisations after fitting the GPD. The region where a straight line is recorded implies a good fit for the GPD. 